\title{
LATERAL HYPOTHALAMIC MODULATION OF THE GUSTATORY-SALIVARY REFLEX IN RATS ${ }^{1}$
}

\author{
RYUJI MATSUO ${ }^{2}$ AND KIYOSHI KUSANO ${ }^{3}$
}

Neurophysiology Laboratory, Department of Biology, Illinois Institute of Technology, Chicago, Illinois 60616

Received June 27, 1983; Revised November 29, 1983; Accepted January 3, 1984

\begin{abstract}
It is well recognized that the basic mechanism for the gustatory-salivary reflex is located in the lower brainstem and that suprabulbar structures possibly influence this mechanism. This study is designed to evaluate the neurophysiological mechanism underlying the effect of the lateral hypothalamic area (LHA) on the bulbar gustatory-salivary reflex. Submandibular salivary secretion and the electrical activity of the preganglionic parasympathetic fibers innervating the submandibular and sublingual glands were recorded in anesthetized rats. Stimulation of the tongue with high concentrations of chemical solutions $(1 \mathrm{M}$ and $2 \mathrm{M} \mathrm{NaCl}$ and $0.01 \mathrm{M}$ and $0.05 \mathrm{M} \mathrm{HCl}$ ) and/or pinching the tongue with a small clamp induced a profuse salivary secretion ( 3 to $28.5 \mu \mathrm{l} / 5 \mathrm{~min}$ ) recorded from a unilateral submandibular gland. The preganglionic fibers consisted of three types: taste-related fibers, which increased their firing rate by taste stimuli; pinch-related fibers, which increased their firing rate by pinching; and unidentified fibers, which did not respond to taste or pinching stimulations of the tongue. Electrical stimulation of the ipsilateral LHA caused the secretion of a small amount of saliva $(1.5 \mu \mathrm{l} / 5 \mathrm{~min})$, and it appeared that taste-related fibers more often received polysynaptic connections from the LIIA than other types of fibers. Conditioning stimulation of the LHA increased the magnitude of impulse discharges by the test stimulation of the tongue in the taste-related fibers. Therefore, the results of this study demonstrate that the LHA enhances the activity of the gustatory-salivary reflex in the lower brainstem.
\end{abstract}

Salivary secretion is reflexly induced by application of a taste solution into the mouth not only in conscious animals but also in anesthetized decerebrate ones. Therefore, the fundamental structures for the gustatory-salivary reflex are considered to be located in the lower brainstem. In this connection, a number of studies have indicated that the primary parasympathetic center for salivary secretion, the superior and inferior salivatory nuclei, exists in the lateral reticular formation in the medulla (see Torvik, 1957; Hiura, 1977; Chibuzo and Cummings, 1980; Matsuo et al., 1980), and that parasympathetic activity mainly controls the volume of saliva during reflex salivary secretion (see Emmelin, 1967; Garrett and Thulin, 1975; Gjörstrup, 1980).

It has long been suggested that the higher levels of the central nervous system influence the medullary secretory center. Noteworthy in this regard, electrical stimulation of the LHA, which is known to contain a feeding center,

\footnotetext{
${ }^{1}$ This work was supported in part by National Institutes of Health Grant NS 12275.

${ }^{2}$ Present address: Department of Oral Physiology, Osaka University Faculty of Dentistry, 18 Yamadaoka, Suita, Osaka 565, Japan.

${ }^{3}$ To whom correspondence should be addressed.
}

elicits organized feeding behavior accompanied with salivation (see Baum, 1945; Hess, 1956; Emmelin, 1967). From recent neuroanatomical studies in rats (Saper et al., 1976; Roberts, 1980; Hosoya and Matsushita, 1981), numerous projections are observed from the LHA to the bulbar autonomic centers, i.e., solitary nucleus, parabrachial nucleus, and nucleus reticularis parvocellularis (part of this nucleus corresponds to salivatory nuclei). These nuclei participate in, or are closely related to, the gustatory-salivary reflex arc in the lower brainstem. Although these behavioral and anatomical findings strongly suggest that the gustatory-salivary reflex is under the control of the LHA, modulatory effects from the LHA upon the gustatory-salivary reflex have not as yet been electrophysiologically demonstrated.

In the present study, in order to examine the effect of the LHA upon the gustatory-salivary reflex, salivary responses in the submandibular and sublingual glands of anesthetized rats were analyzed, because the gustatory information from the anterior part of the tongue predominantly activates the submandibular gland rather than the parotid gland (Miller, 1913; Miyake, 1969). To evaluate the activity of the salivary gland, the amounts of salivary secretion and neural discharge in pregan- 
glionic parasympathetic fibers innervating the submandibular or sublingual glands were recorded in response to electrical stimulation of the LHA and to gustatory, thermal, and mechanical stimulation of the tongue.

\section{Materials and Methods}

Forty-one male Sprague-Dawley rats weighing 290 to $410 \mathrm{gm}$ were used. The animals were anesthetized by intraperitoneal injection of a mixture of $\alpha$-chloralose (50 $\mathrm{mg} / \mathrm{kg})$ and urethane $(500 \mathrm{mg} / \mathrm{kg})$. The trachea was cannulated, and both sides of the superior cervical ganglia were removed for sympathetic denervation of the salivary glands. After implanting an LHA stimulating electrode, the head of the animal was stabilized on its left or right side for recording salivary secretion and activity of preganglionic fibers. When the blinking reflex reappeared, recording of the salivary reflex was started. If necessary, additional small doses of urethane were supplied via a cannula placed in the femoral vein.

The procedures for stimulating the LHA and for stimulating the oral region were essentially the same as in the preceding paper (Matsuo et al., 1984). However, for taste stimulation of the tongue, the following reagent grade chemicals dissolved in distilled water were employed: $0.5 \mathrm{M}$ and $1 \mathrm{M}$ sucrose, $1 \mathrm{M}$ and $2 \mathrm{M} \mathrm{NaCl}, 0.01$ $\mathrm{M}$ and $0.05 \mathrm{M} \mathrm{HCl}$, and $0.02 \mathrm{M}$ and $0.05 \mathrm{M}$ quinine- $\mathrm{HCl}$. These high concentrations of chemicals were necessary to obtain sufficient salivary secretion and efferent nerve discharges of the preganglionic parasympathelic fibers in the anesthetized animals. In addition to a gentle mechanical stimulation by stroking the tongue surface with a small glass rod, a far stronger mechanical stimulation was applied by pinching the lips or the tongue with a small clamp.

Recording of salivary flow. Submandibular salivary secretion was recorded from the ipsilateral side of the LHA stimulating electrode. The duct of the submandibular salivary glands was cannulated with polyethylene tubing with internal and external diameters of 0.3 and $0.7 \mathrm{~mm}$, respectively. The point of insertion of the tubing was always proximal to the intersection between the chordalingual nerve and the duct. When the tubing had been inserted into the submandibular duct the passage was tested by electrical stimulation of the chorda-lingual nerve $(5 \mathrm{~V}, 0.5 \mathrm{msec}, 20 \mathrm{~Hz})$. The other end of the cannula was connected horizontally to a $100-\mu l$ glass pipette for measuring the amount of salivary secretion. During each measuring period (usually $5 \mathrm{~min}$ ), a test solution (about $3 \mathrm{ml}$ ) was applied every $1 \mathrm{~min}$. In the case of electrical stimulation, touch and/or pinching, each stimulus was applied for 15 sec every $1 \mathrm{~min}$.

Recording of preganglionic secretory nerve discharges. The chorda-lingual nerve near the intersection with the ducts of the submandibular and sublingual glands gives off several branches of preganglionic parasympathetic fibers to innervate the salivary glands. These nerve bundles run along the ducts until they reach the submandibular ganglia which are most abundant on the surface of the ducts near the hilus of the glands. The activity of fine strands of the preganglionic efferent fibers was recorded from the central cut ends of the branches at a point before reaching the ganglia. A monopolar platinum wire electrode ( $100 \mu \mathrm{m}$ in diameter) was used for recording nerve discharges from a functionally single fiber or from a few fibers. A small chloridized $\mathrm{Ag} / \mathrm{AgCl}$ plate was placed on surrounding tissues as an indifferent electrode. The nerve impulses were amplified with a Grass P 15 ac preamplifier and displayed on a storage oscilloscope, Tektronix 5113. The amplified potentials were stored by a Vetter Instrumentation recorder model B and later photographed. Some of the stored data led into a spikeamplitude discriminator, and then the rate of spike discharges was determined by a rate/interval monitor (Frederick Hare \& Co.).

\section{Results}

Salivary secretion by oral and hypothalamic stimulation. Salivary secretion from the mandibular gland was elicited by electrical stimulation of the LHA at the level of the ventromedial nucleus in sympathetically decentralized rats. However, the flow of saliva by LHA stimulation was relatively smaller than that by stimulation applied to the oral region. Figure 1 shows the volume of salivary secretion induced by different stimuli of the oral region and the LHA. Each value represents the mean \pm $\mathrm{SD}(n=5)$ for $5 \mathrm{~min}$ of recording. On oral stimulation, the volume of reflex salivation depended on the character of the stimulation applied. Thermal stimulation and stroking the surface of the tongue with a glass rod evoked little salivation, while high concentrations of chemicals (such as $2 \mathrm{M} \mathrm{NaCl}, 0.01 \mathrm{M}$ and $0.05 \mathrm{M} \mathrm{HCl}$ ) induced a copious salivation ranging from 4 to $18 \mu \mathrm{l} / 5 \mathrm{~min}$. Pinching of the tongue or the lips, noxious mechanical stimulation, always induced more copious salivary secretion (25 to $44 \mu \mathrm{l} / 5 \mathrm{~min}$ ) than any other kind of stimulus. On LHA electrical stimulation, a mean value of $1.5 \mu \mathrm{l} / 5 \mathrm{~min}$ of saliva was elicited at $5-\mathrm{Hz}$ frequency. This value was about $22 \%$ of the salivation induced by electrical stimulation of the anterior part of the tongue at $5-\mathrm{Hz}$ frequency.

Activities of the preganglionic parasympathetic fibers. Multiunit neural activity was recorded from one of the branches of the preganglionic parasympathetic fibers innervating the submandibular and sublingual glands. As shown in Figure 2, electrical stimulation of the tongue elicited strong activity lasting from 20 to $140 \mathrm{msec}$, while electrical stimulation of the LHA induced a smaller response lasting from 60 to $160 \mathrm{msec}$. In both cases, ipsilateral stimulation was always more effective than contralateral stimulation. When the number of impulses evoked by ipsilateral tongue stimulation $(27.8 \pm 2.4$ impulses/stimulus, $n=10$ ) was taken as the standard $(100 \%)$, stimulation of the contralateral tongue, the ipsilateral LHA, and the contralateral LHA evoked 57.5, 44.5 , and $4.0 \%$ of the standard, respectively.

Electrical activity of functionally single fibers and/or a few strands of the preganglionic fibers was recorded, and a total of 54 unitary activities were sampled. They were classified into three groups according to their responsiveness to oral stimulation. Twenty $(38 \%)$ of 54 fibers increased their firing rate to taste stimuli-tasterelated fibers. These fibers showed spontaneous firing of $0.22 \pm 0.17$ impulses/sec (mean $\pm \mathrm{SD}, n=20$ ), and they responded to electrical stimulation applied to the tongue 


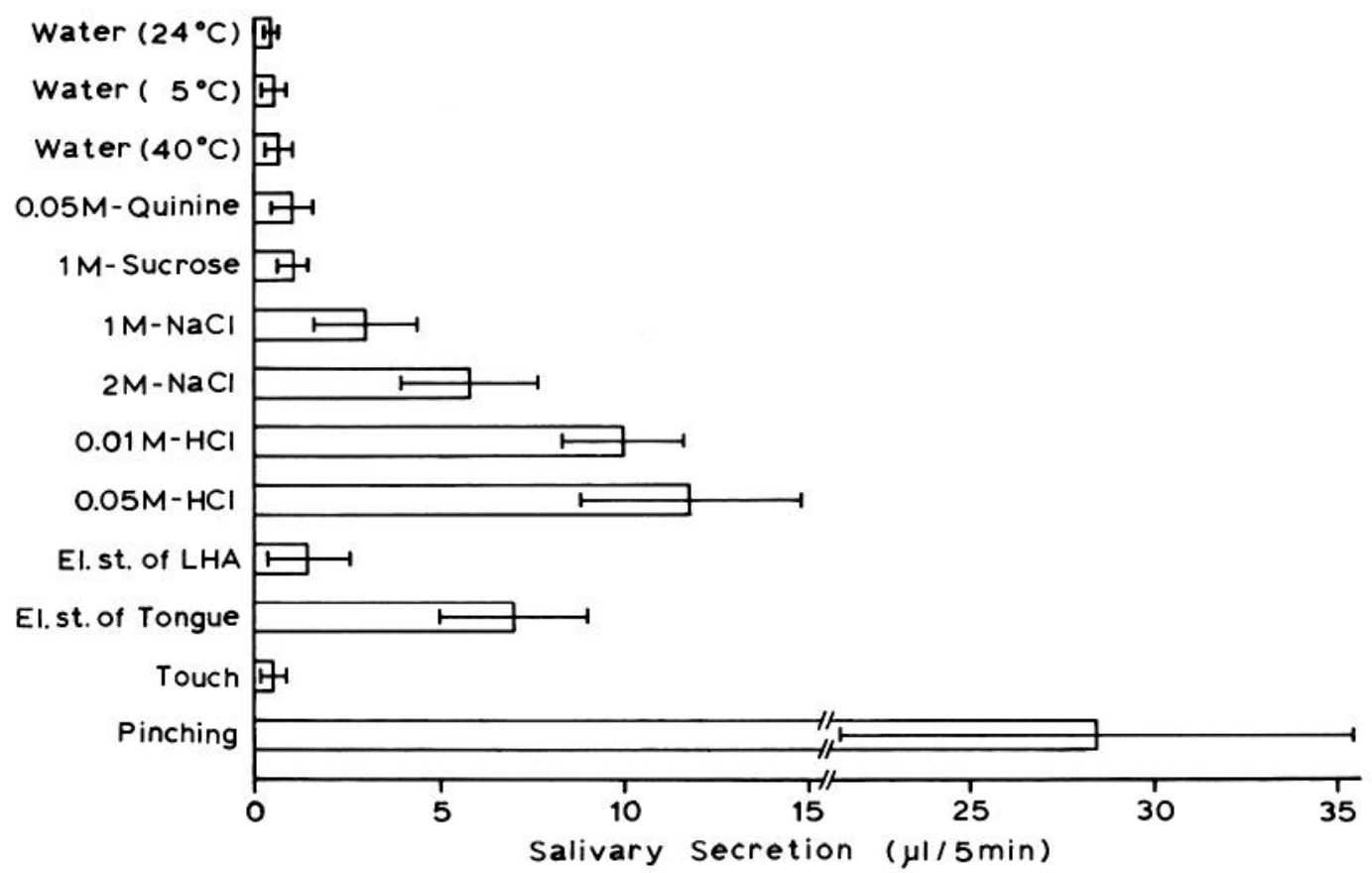

Figure 1. Salivary secretion, recorded from a unilateral submandibular gland, induced by stimulation of the tongue and the LHA. Bars are mean \pm SD values for 5-min recordings obtained from five different animals. Large amounts of salivary secretion were evoked by relatively strong taste stimuli $(2 \mathrm{M} \mathrm{NaCl}, 0.01 \mathrm{M}$ and $0.05 \mathrm{M} \mathrm{HCl})$ and/or pinching applied to the oral region. Repetitive electrical stimulation of the LHA at $5 \mathrm{~Hz}$ induced a small amount of saliva.

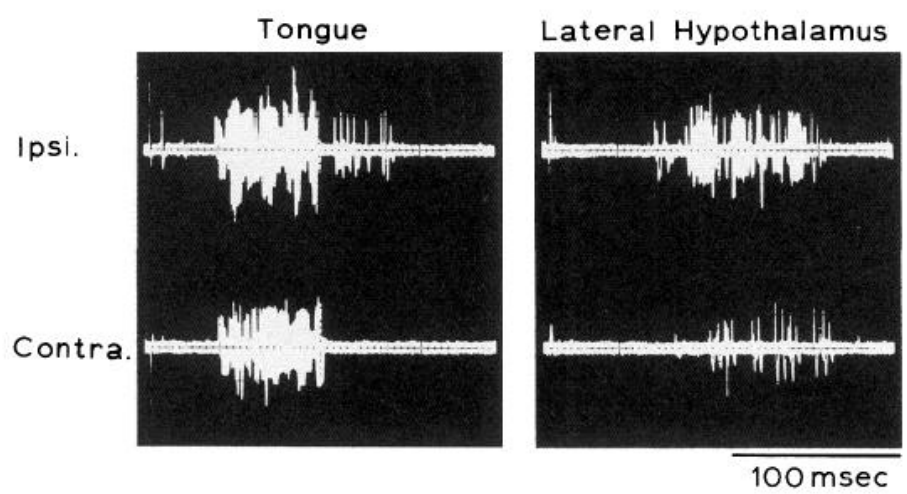

Figure 2. Multiunit neural responses recorded from the preganglionic parasympathetic fibers innervating the submandibular and sublingual glands. Each recording shows 10 superimposed sweeps at $1-\mathrm{Hz}$ frequency. Left column, responses elicited by tongue stimulation. A large response with a latency of about 20 to $140 \mathrm{msec}$ was produced by ipsilateral (Ipsi.) tongue stimulation rather than by contralateral (Contra.) stimulation. Right column, responses elicited by LHA stimulation. About $30 \%$ of the magnitude of the response to ipsilateral tongue stimulation was obtained by ipsilateral LHA stimulation with a latency of about 50 to $150 \mathrm{msec}$. Contralateral stimulation induced only several spike discharges.

with a latency of $42.4 \pm 23.5 \mathrm{msec}(n=20)$. Eleven (20\%) fibers, which were not responsive to taste stimuli, increased their firing rate when the tongue or lips were pinched-pinch-related fibers. These fibers showed spontaneous firing of $0.24 \pm 0.24$ impulses/sec $(n=11)$, and their latency to electrical stimulation of the tongue was $51.7 \pm 15.9 \mathrm{msec}(n=11)$. Nineteen $(35 \%)$ fibers were responsive to electrical stimulation of the tongue but were not responsive to the thermal, tactile, taste and pinching stimuli employed in this experiment-unidentified fibers. This type of fiber showed a higher rate of spontaneous firing $(0.50 \pm 0.42 \mathrm{impulses} / \mathrm{sec}, n=19)$ and a shorter latency to electrical stimulation of the tongue $(11.8 \pm 3.2 \mathrm{msec}, n=19)$. Recordings of these three types of fibers are shown in Figure 3.

In addition to the three types of fibers, four (7\%) showed characteristics of first-order afferent fibers rather than of preganglionic efferent fibers. These fibers showed an all-or-none response with a constant latency of $4.2 \pm 1.7 \mathrm{msec}(n=4)$ and followed $100-\mathrm{Hz}$ repetitive electrical stimulation of the tongue. They did not respond to LHA stimulation. As shown in Figure 4, one fiber of four was responsive to taste stimuli $(A)$ and three to stroking the tongue surface $(B)$. Incidentally, none of the preganglionic efferent fibers (taste-related, pinchrelated, and unidentified) responded to light mechanical stimulation by stroking the tongue surface.

Among three types of the preganglionic parasympathetic fibers $(n=50), 28(56 \%)$ were also driven by electrical stimulation applied to the ipsilateral LHA; i.e., 14 $(70 \%)$ of 20 taste-related, $6(55 \%)$ of 11 pinch-related, and $8(42 \%)$ of 19 unidentified fibers were responsive. The responsiveness to LHA stimulation was relatively variable as compared with that to tongue stimulation. Namely, when electrical stimulation was applied to the LHA at $1-\mathrm{Hz}$ frequency, each electrical shock induced a variable number of impulses (up to three) and occasionally no impulse. These impulses occurred during a period of $21.6 \pm 18.1 \mathrm{msec}$ (mean $\pm \mathrm{SD}, n=28$ ) after the onset of stimulation, ranging from 6 to $72 \mathrm{msec}$, which were measured from a period between the first and the last impulses in 10 superimposed traces of each fiber. While the number of impulses per stimulus applied to the 
A

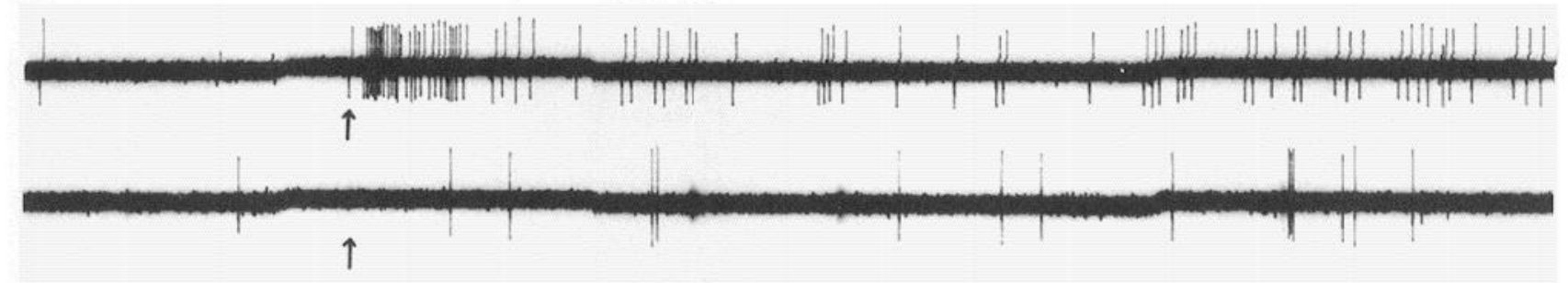

A

b
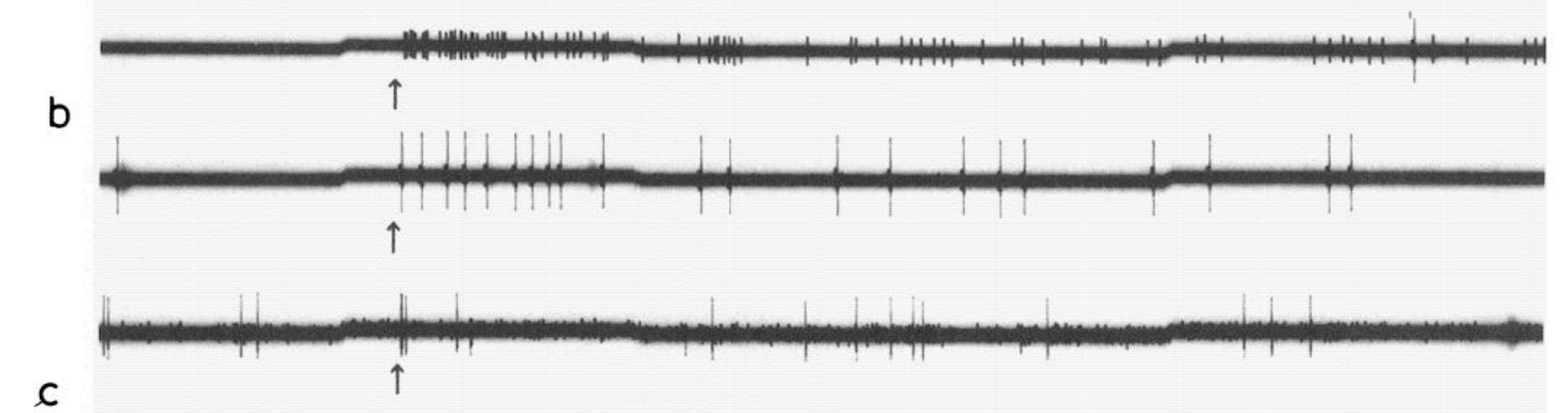

C

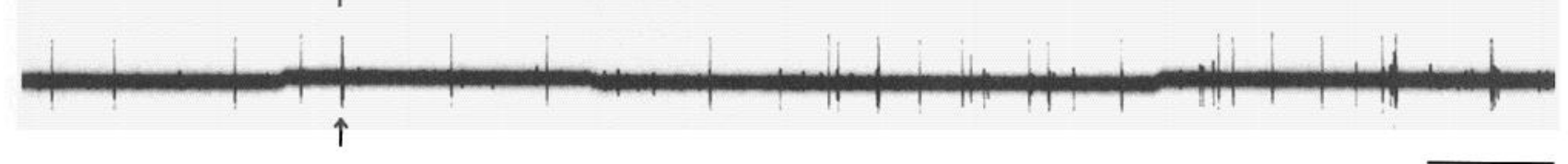

B

a

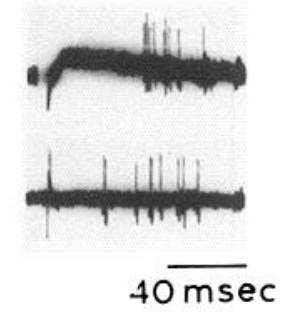

b
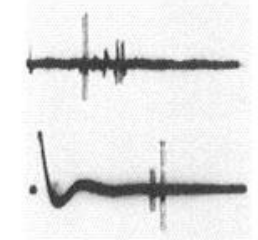

$80 \overline{\mathrm{msec}}$

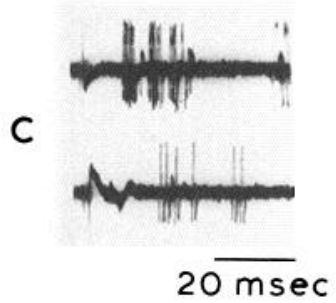

$1 \mathrm{Sec}$

Figure 3. Examples of taste-related $(A a, B a)$, pinch-related $(A b, B b)$, and unidentified $(A c, B c)$ fibers in the preganglionic parasympathetic fibers. $A a, \mathrm{~A} 0.01 \mathrm{M} \mathrm{HCl}$ solution poured on the tongue at the arrow produced spike discharges in the upper trace, but this fiber did not respond to pinching stimulation applied at the arrow for 5 sec in the lower trace. Ab, Two types of units were recognized. A small unit was responsive to $0.01 \mathrm{M} \mathrm{HCl}$ in the upper trace but not to pinching in the lower trace. A large unit was responsive only to pinching stimulation. Ac, This fiber did not respond either to $0.01 \mathrm{M} \mathrm{HCl}$ or to pinching stimulation, as seen in the upper and lower traces, respectively. Although taste, mechanical (pinching and stroking the tongue surface), and thermal (cool and warm water) stimulations were employed, adequate stimulation for this unit was not found. This type often showed higher spontaneous discharge rates than other types. $B$, Spike discharges driven by electrical stimulation of the tongue (upper traces) and the LHA (lower traces). All traces are composed of five superimposed sweeps. The spike latencies to electrical stimulation of the tongue and the LHA were shorter in unidentified fibers (Bc) than in taste-related (Ba) and/or pinch-related $(B b)$ fibers.

tongue at $1 \mathrm{~Hz}$ was also variable (from one to three), the impulses discharged during a shorter period of $13.9 \pm$ $10.0 \mathrm{msec}(n=54$, range from 1 to $34 \mathrm{msec})$. And Figure 5 shows distribution of the mean latencies of the first impulses in 54 tongue-responsive fibers (upper histogram) and 28 LHA-responsive fibers (lower histogram), which were obtained from averaging the values of 10 trials of each unitary discharge. The mean latencies to LHA stimulation were more widely distributed than those to tongue stimulation.

The mode of impulse discharge in the three types of preganglionic fibers was analyzed by applying repetitive electrical stimulation at varying frequencies. Figure 6 shows the relationship between the frequency of electrical stimulation and the discharge rate measured for 10 sec after the onset of the stimulation. Each plot is the mean $\pm \mathrm{SD}$ of values obtained in six fibers. As indicated by the upper graph in Figure 6, tongue stimulation induced the maximal discharge rate in taste-related and pinch-related fibers at around $10-\mathrm{Hz}$ frequency. The discharge rates at $10 \mathrm{~Hz}$ in taste-related and pinchrelated fibers were $6.9 \pm 2.2$ and $6.8 \pm 3.0$ impulses/sec, respectively. As to unidentified fibers, the maximal discharge rate $(3.8 \pm 1.5$ impulses/sec) was induced at $5-\mathrm{Hz}$ frequency. In the lower graph of Figure 6, electrical stimulation of the LHA, which evoked lower firing rates of discharge than in the same fibers analyzed in the upper graph, is shown. The taste-related and the pinchrelated fibers increased their firing rates at around $5-\mathrm{Hz}$ frequency, and their discharge rates at $5 \mathrm{~Hz}$ were $3.1 \pm$ 1.3 and $1.9 \pm 0.6$ impulses/sec, respectively. However, the unidentified fibers did not show noteworthy changes 


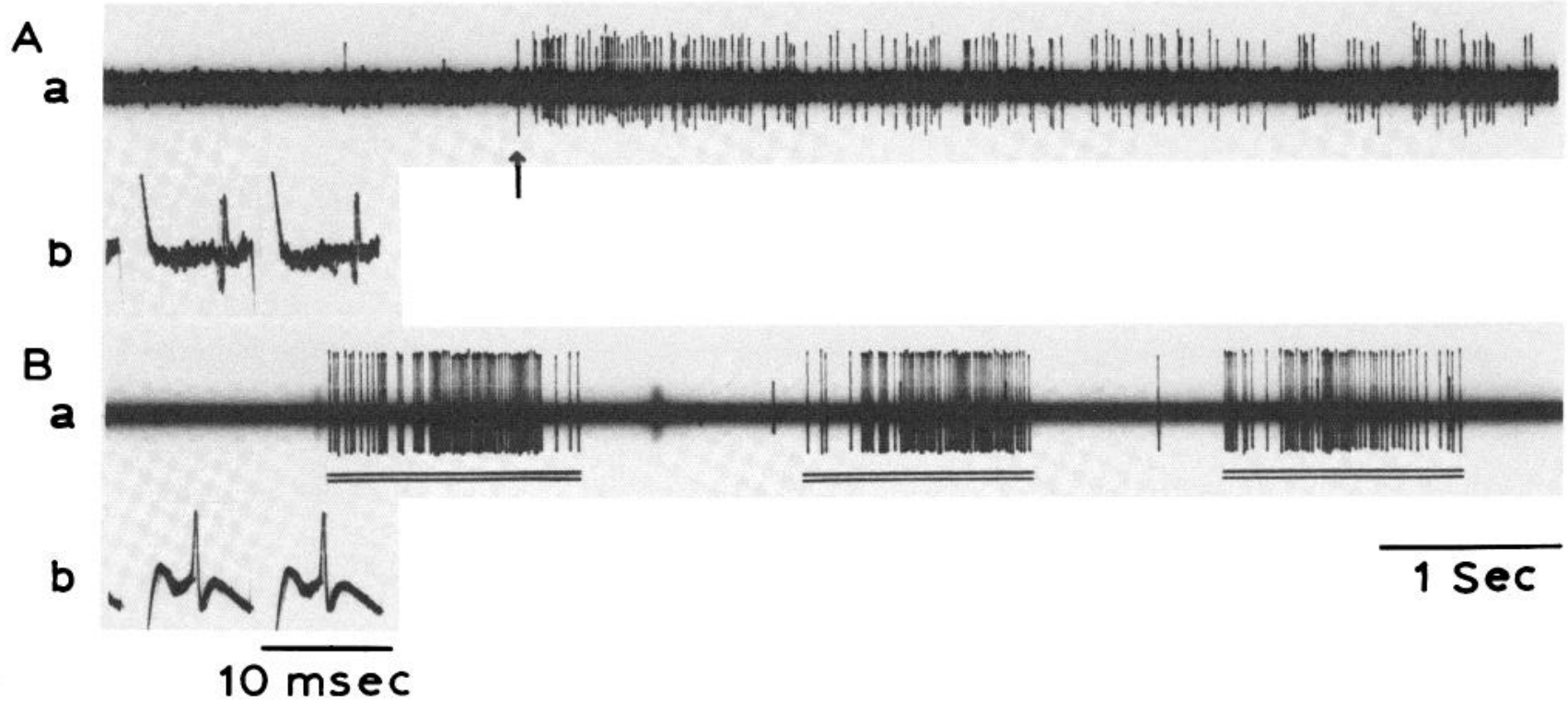

Figure 4. Examples of unitary discharges, which show characteristics of first-order afferent fibers, recorded from branches of the preganglionic parasympathetic fibers. $A a$, This unit responded to $0.01 \mathrm{M} \mathrm{HCl}$ solution applied to the tongue at the $a r r o w$. $B a$, Tonic spike discharges were produced by stroking the surface of the tongue, indicated by double bars. Both units responded to electrical stimulation of the tongue with a constant latency, and they followed $100-\mathrm{Hz}$ repetitive stimulation $(A b, B b)$.

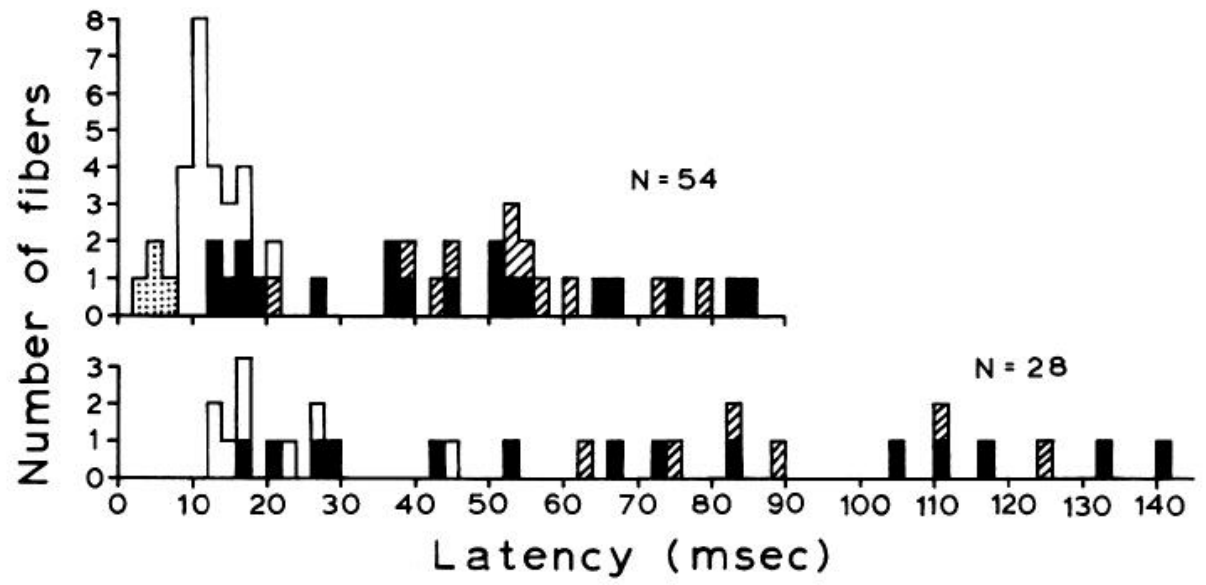

Figure 5. The latency of the impulse discharges evoked by tongue stimulation (upper histogram) and by LHA stimulation (lower histogram). The latency for each fiber was obtained by averaging the values of 10 trials. Solid columns, taste-related fibers; hatched columns, pinch-related fibers; open columns, unidentified fibers; dotted column, "first-order afferent" fibers. Taste-related and pinch-related fibers showed longer latencies than unidentified fibers by both tongue and LHA stimulation.

in their discharge rates by repetitive stimulation of the LHA.

Relationship between salivary flow and neural responses in the preganglionic fibers. The relationship between the magnitude of efferent discharges and the volume of salivary outflow from the submandibular gland in response to 11 different stimuli applied to the tongue was examined and is shown in Figure 7. The ordinate of the graph in this figure indicates the mean volume of salivary secretion obtained from five animals and shows the same data as in Figure 1. The abscissa indicates the mean number of impulses per second obtained from five tasterelated (solid circles) and five pinch-related (open circles) fibers. The magnitude of impulse discharge for each fiber was measured for $10 \mathrm{sec}$ after the onset of stimulation and was expressed as impulses per second. The mean volume of salivary flow correlated significantly with the mean number of impulses in taste-related and pinchrelated fibers ( $r=0.87, p<0.001$, the Student's $t$ test), whereas salivation did not correlate with the mean impulses in unidentified fibers $(r=0.14, p>0.5$, the Student's $t$ test).

Influence of LHA activity on the reflex discharges in the preganglionic parasympathetic fibers. The effect of LHA stimulation on the reflex discharges induced by tongue stimulation was examined in the three types of 


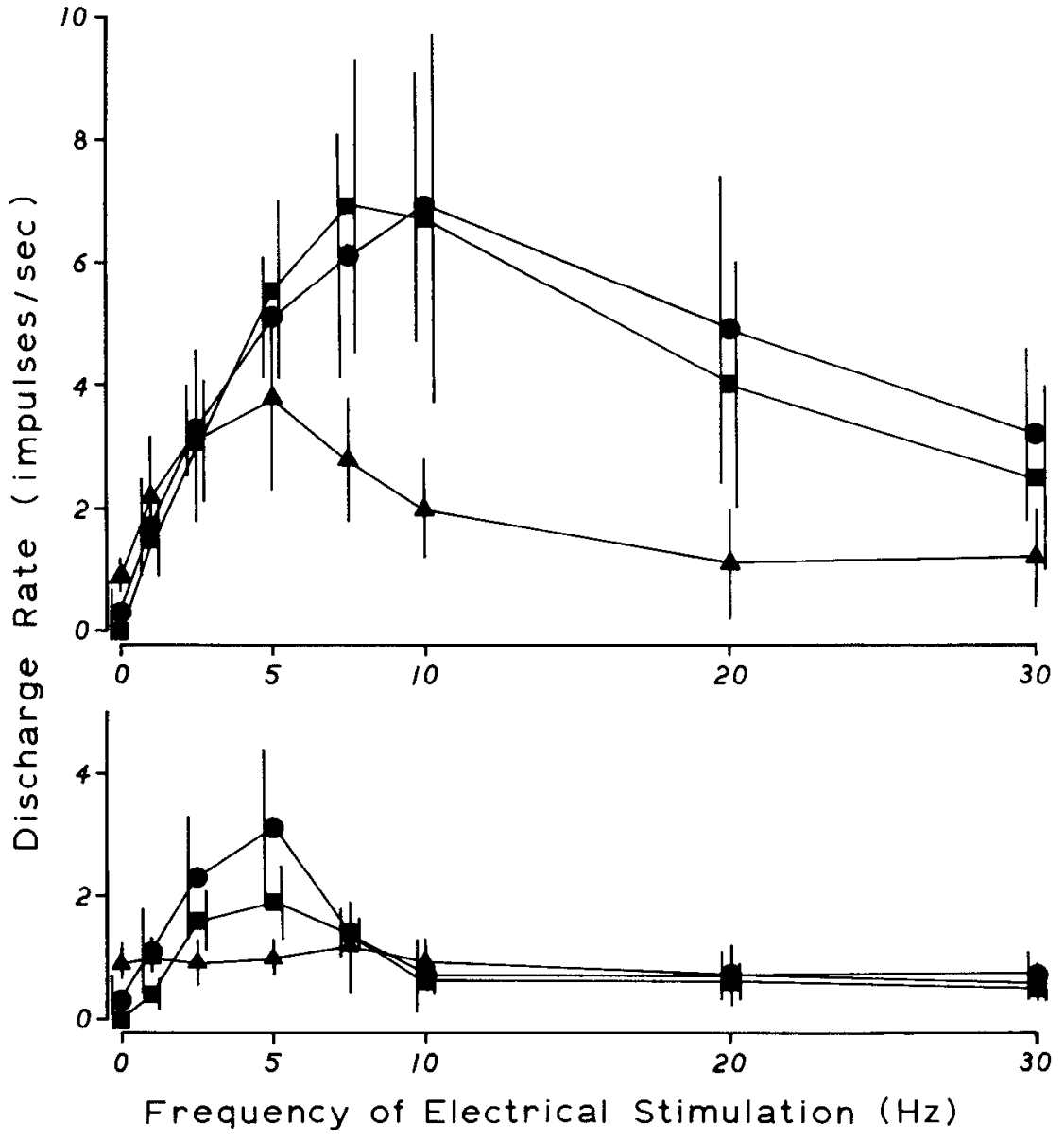

Figure 6. Relationship between the discharge rate of the preganglionic parasympathetic fibers and the frequency of repetitive electrical stimulation applicd to the tongue (upper graph) and the LHA (lower graph). The discharge rate was measured in taste-related $(\boldsymbol{O})$, pinch-related $(\boldsymbol{\square})$, and unidentified $(\boldsymbol{\Delta})$ fibers during stimulation for $10 \mathrm{sec}$. Each value is the mean $\pm \mathrm{SD}(n=6)$.

preganglionic fibers when a conditioning stimulus to the LHA was applied prior to a test stimulus to the tongue at varying intervals. Figure 8 represents examples of the time course of the effects of the conditioning stimulation in taste-related (solid line), pinch-related (broken line), and unidentified (dotted line) fibers. At conditioning-test intervals of 10 to $150 \mathrm{msec}$, the number of impulses per stimulus in taste-related and pinch-related fibers increased by about 20 to $50 \%$ of their control levels. However, the unidentified fibers reduced the number of impulses per test stimulus by about 70 to $80 \%$ of their control level at conditioning-test intervals of $50 \mathrm{msec}$ and then gradually recovered from this suppression as the conditioning-test intervals exceeded about $200 \mathrm{msec}$.

\section{Discussion}

Reflex salivation at the level of the lower brainstem. The preganglionic parasympathetic fibers innervating the submandibular and sublingual glands could be classified into three types (taste-related, pinch-related, and unidentified fibers) according to their responsiveness to stimulation of the anterior part of the tongue. That is, taste-related and pinch-related fibers increased their firing rates by application of taste solutions and pinching, respectively. Unidentified fibers did not respond to the taste, thermal, tactile, or painful mechanical stimulations employed in this experiment. Since the volume of reflex salivation correlated significantly with the magnitude of responses in taste-related and pinch-related fibers, these two types of fibers are considered to be parasympathetic secretory fibers which control the volume of saliva by affecting the activity of secretory cells and/or myoepithelial cells through postganglionic fibers. On the other hand, the function of unidentified fibers is not yet clear; however, there is a possibility that some of these fibers could control the functions of the ducts or blood vessels in the salivary glands, because of the following notions. In view of the morphology of submandibular or sublingual glands of rats (Snell and Garrett, 1957; Snell, 1960; Garrett, 1974), parasympathetic nerve endings were identified around ducts and blood vessels as well as around acini. Also it is generally accepted that the ducts of salivary glands control ion secretion (Schneyer et al., 1972) and that parasympathetic stimulation of salivary glands results in vasodilation and secretion (Emmelin, 1967).

Taste-related and pinch-related fibers showed relatively long latencies to electrical stimulation applied to the tongue, i.e., $42.4 \pm 23.5 \mathrm{msec}(n=20)$ for tasterelated and $51.7 \pm 15.9 \mathrm{msec}(n=11)$ for pinch-related 


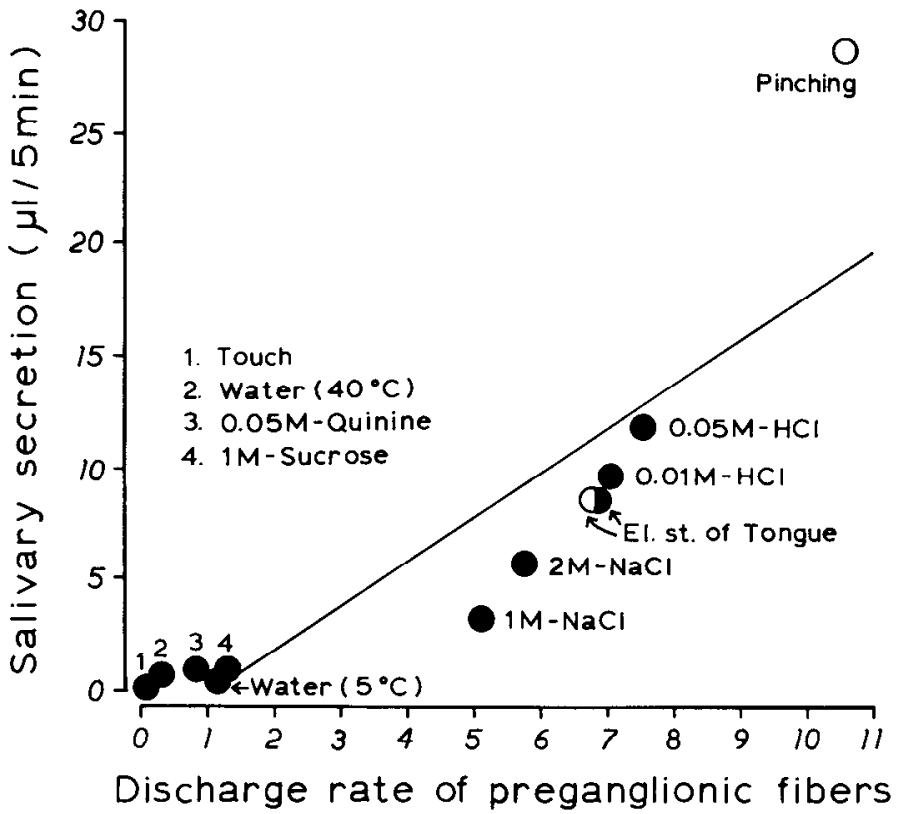

(impulses /sec)

Figure 7. Relationship between the volume of salivary secretion, recorded from a unilateral submandibular gland, and the rate of impulse discharges in taste-related $(\Theta)$ and pinch-related (O) fibers. Salivary secretion and impulse discharges were recorded from different animals. Each plot represents the mean value for salivary secretion in five animals, for five taste-related and for five pinch-related fibers. The volume of salivary secretion correlated significantly with the magnitude of the response in taste-related and pinch-related fibers. Regression line: $Y=$ $1.98 X-2.19(r=0.87, p<0.001, n=12)$. fibers. Such a long latency of response was also recorded in rats which were decerebrated at the precollicular level of the midbrain in our preliminary experiments and in previous reports using rabbits (Kawamura and Yamamoto, 1978; Kawamura et al., 1982; Matsuo et al., 1982). Therefore, such a long latency of reflex activity seems to be taken up in the salivary reflex arc of the lower brainstem. In the present study, application of taste and pinching stimulations to the tongue were effective in evoking a copious flow of saliva, and two types of parasympathetic secretory fibers (taste-related and pinchrelated fibers) were recognized. These findings imply that gustatory and mechanical information do not converge on a common superior salivatory neuron, and that two different kinds of pathways exist in the salivary reflex arc of the lower brainstem. For taste-related fibers, gustatory afferents possibly come from the nucleus tractus solitarius and/or the parabrachial nucleus to the superior salivatory nucleus. Those two nuclei are relay areas of the gustatory afferents in the lower brainstem (Norgren and Leonard, 1973; Norgren, 1978). For pinch-related fibers, mechanical stimulation reaches the superior salivatory nucleus through the trigeminal sensory nuclear complex (see Darian-Smith, 1966; Khayyat et al., 1975; Sessle and Greenwood, 1976; Kawamura and Yamamoto, 1977).

The first-order afferent discharges were recorded from branches of the preganglionic parasympathetic fibers. Although explanation of the functional significance of these fibers is beyond the scope of the present study, these fibers may not be concerned with controlling the volume of saliva, because little salivary secretion was

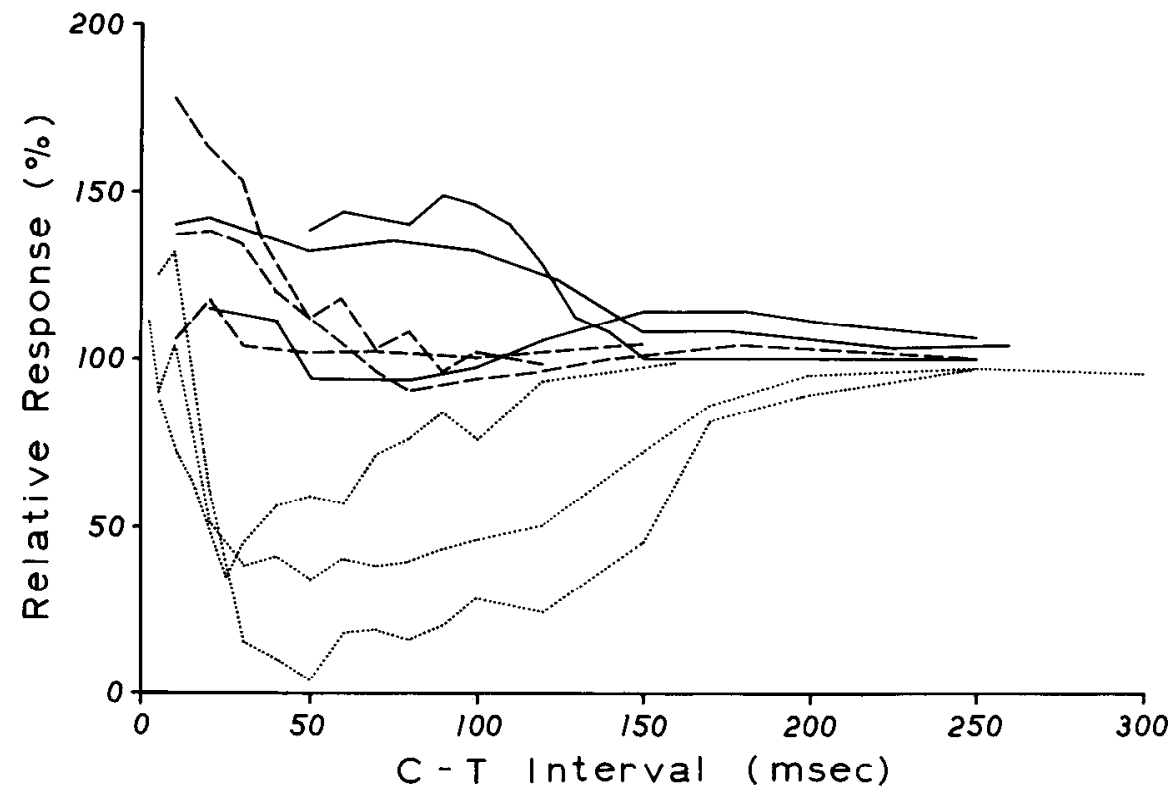

Figure 8. Time course of the effect of conditioning LHA stimulation upon reflex discharges of the preganglionic parasympathetic fibers evoked by test stimulation applied to the tongue. Relative response is expressed as a percentage on the ordinate, when the mean number of impulses induced by 10 test stimuli in the presence of the conditioning stimuli was divided by that induced by 10 control tongue stimuli at each conditioning-test interval. Taste-related (solid lines) and pinch-related (broken lines) fibers show facilitatory effects at conditioning-test intervals of about 10 to $150 \mathrm{msec}$, whereas unidentified (dotted lines) fibers show suppressive effects at about 10 to $200 \mathrm{msec}$. 
evoked by stroking the surface of the tongue in spite of producing vigorous impulse discharges in such fibers by the same mechanical stimulation. The discharge rates of these fibers were very high: 60 to 80 impulses/sec by mechanical stimulation and about 40 impulses/sec by $0.01 \mathrm{M} \mathrm{HCl}$ solution. According to an electrophysiological study of the submandibular ganglion cells of rats (Suzuki and Sakada, 1972), the maximum frequencies permitting transmission of impulses at synapses of the ganglion cells were 30 to $60 \mathrm{~Hz}$ in cells innervated by B fibers and $20 \mathrm{~Hz}$ in cells innervated by $\mathrm{C}$ fibers. Therefore, even if these fibers have synaptic connections with the submandibular ganglion cells, the rate of the first-order afferent discharges is beyond the capacity of synaptic transmission of the ganglion cells and salivary secretion will not be effectively induced.

In connection with discharge rate of secretory fihers, Emmelin and Holmberg (1967) showed in dogs that the amount of salivary secretion by food ingestion was comparable to the salivary flow evoked by the electrical stimulation of the preganglionic fibers at 4 to 8 pulses/ sec, and that of the very copious secretion by ingesting rejectable substances was comparable to the flow produced by a stimulation rate of 10 to 30 pulses/sec. Suzuki (1981) reported that the submandibular ganglion cells of rats reflexly discharged at 10 to 12 spikes/sec on exposing the rat to a high ambient temperature of $38^{\circ} \mathrm{C}$ and by applying gustatory stimuli to the tongue. Similarly, low discharge rates of 2 to $5 \mathrm{impulses} / \mathrm{sec}$, in response to four basic taste solutions at physiological concentrations, were recorded from the rabbit submandibular preganglionic parasympathetic fibers (Kawamura and Yamamoto, 1978; Kawamura et al., 1982). Therefore, the fairly low rate of discharge in taste-related and pinch-related fibers observed here $(7.1 \pm 3.5$ impulses/sec, $n=5$, by $0.01 \mathrm{M} \mathrm{HCl}$ solution and 10.5 \pm 3.4 impulses/sec, $n=5$, by pinching of the tongue) seems to be near the firing rate when animals are ingesting foodstuffis or rejecting unpalatable substances.

Modulatory effects from the LHA upon the reflex salivation of the lower brainstem. When the ipsilateral LHA was electrically stimulated, about one-half of the preganglionic parasympathetic fibers responded. The response to LHA stimulation is presumably more polysynaptic activation than that obtained from tongue stimulation because of the following properties. As shown in Figure 5 , the latency of response to LHA stimulation was relatively longer and more widely scattered than that of the response to tongue stimulation. The ability to follow repetitive electrical stimulation was higher in tongue stimulation than in LHA stimulation (Fig. 6). In addition, observation of the discharge pattern to each electrical shock reveals multiple discharges occurring for a longer period ( $21.6 \pm 18.1 \mathrm{msec}, n=28)$ on LHA stimulation than on tongue stimulation $(13.9 \pm 10.0 \mathrm{msec}, n$ $=54$ ).

Among the LHA-responsive fibers, $50 \%, 29 \%$, and $21 \%$ were taste-related, unidentified, and pinch-related fibers, respectively. This fact suggests that taste-related fibers dominantly receive polysynaptic activation from the LHA and that a small amount of salivary secretion induced by LHA stimulation per se was due to activity in taste-related fibers. As already discussed, the bulbar salivary reflex arc for taste-related fibers may involve the nucleus tractus solitarius and the parabrachial nucleus. These nuclei, the dorsal raphe nucleus, and the nucleus reticularis parvocellularis (part of this nucleus corresponds to salivatory nuclei) are projected from the LHA as revealed by neuroanatomical studies (Saper et al., 1976; Hosoya and Matsushita, 1981). Therefore, the LHA may activate the above-mentioned nuclei, which results in polysynaptic activation of the superior salivatory nucleus.

By applying conditioning stimulation of the LHA prior to the test stimulation of the tongue, different modulatory effects were observed among taste-related, pinchrelated, and unidentified fibers. In taste-related and pinch-related fibers, conditioning stimulation of the LHA increased the number of impulses per test stimulus by 20 to $50 \%$ of their control level for a period of 10 to $150 \mathrm{msec}$; whereas in unidentified fibers, the number of impulses per test stimulus was reduced by about 70 to $80 \%$ of their control level for a $30-$ to $150-\mathrm{msec}$ period. The facilitatory effect in taste-related fibers is similar to that in the gustatory neurons of the nucleus tractus solitarius explored in the preceding paper (Matsuo et al., 1984). These results suggest that the reactivity of the gustatory-related autonomic centers in the lower brainstem is increased by the LHA activity. Although unidentified fibers showed a similar suppressive effect to those of mechanical and thermal neurons of the nucleus tractus solitarius (Matsuo et al., 1984), unidentified fibers may not receive inputs from the mechanical or thermal neurons of the nucleus tractus solitarius since they did not respond to gentle mechanical or thermal stimulation of the tongue.

\section{References}

Baum, W. (1945) Speichelfluss als Symptom elktrischer Reizung im Zishenhirn und den angrenzenden Gebieten. Helv. Physiol. Pharmacol. Acta 3: 21-40.

Chibuzo, G. A., and J. F. Cummings (1980) Motor and sensory centers for the innervation of mandibular and sublingual salivary glands: A horseradish peroxidase study in the dog. Brain Res. 189: 301-313.

Darian-Smith, I. (1966) Neural mechanisms of facial sensation. Int. Rev. Neurobiol. 9: 301-395.

Emmelin, N. (1967) Nervous control of salivary glands. In Handbook of Physiology. Sect. 6: Alimentary Canal II, pp. 595-632, American Physiological Society, Washington, D. C.

Emmelin, N., and J. Holmberg (1967) Impulse frequency in secretory nerves of salivary glands. J. Physiol. (Lond.) 191: $205-214$.

Garrett, J. R. (1974) Innervation of salivary glands; morphological considerations. In Secretory Mechanisms of Exocrine Glands, N. A. Thorn and O. H. Petersen, eds., pp. 17-27, Munksgaard, Copenhagen.

Garrett, J. R., and A. Thulin (1975) Changes in parotid acinar cells accompanying salivary secretion in rats on sympathetic or parasympathetic nerve stimulation. Cell Tissue Res. 159: 179-193.

Gjöstrup, P. (1980) Parotid secretion of fluid and amylase in rabbits during feeding. J. Physiol. (Lond.) 309: 101-116.

Hess, W. R. (1956) Hypothalamus und Thalamus, Thieme, Stuttgart.

Hiura, T. (1977) Salivatory neurons innervate the submandibular and sublingual glands in the rat: Horseradish peroxidase study. Brain Res. 137: 145-149. 
Hosoya, Y., and M. Matsushita (1981) Brainstem projections from the lateral hypothalamic area in the rat, as studied with autoradiography. Neurosci. Lett. 24: 111-116.

Kawamura, Y., and T. Yamanolo (1977) Salivary secretion to noxious stimulation of the trigeminal area. In Pain in the Trigeminal Region, D. J. Anderson and B. Matthews, eds., pp. 395-404, Elsevier, Amsterdam.

Kawamura, Y., and T. Yamamoto (1978) Studies on neural mechanisms of the gustatory-salivary reflex in rabbits. J. Physiol. (Lond.) 285: 35-47.

Kawamura, Y., R. Matsuo, and T. Yamamoto (1982) Analysis of reflex responses in preganglionic parasympathetic fibres innervating submandibular glands of rabbits. J. Physiol. (Lond.) 322: 241-255.

Khayyat, G. F., Y. J. Yu, and R. B. King (1975) Response patterns to noxious and non-noxious stimuli rostral trigeminal relay nuclei. Brain Res. 97: 47-60.

Matsuo, R., T. Yamamoto, and Y. Kawamura (1980) Morphological and functional evaluation of the superior salivatory nucleus in rabbits. Exp. Neurol. 68: 147-157.

Matsuo, R., T. Yamamoto, and Y. Kawamura (1982) Responses of salivatory neurons in the medulla oblongata of rabbit. Jpn. J. Physiol. 32: 309-313.

Matsuo, R., N. Shimizu, and K. Kusano (1984) Lateral hypothalamic modulation of oral sensory afferent activity in nucleus tractus solitarius neurons of rats. J. Neurosci. 4: 12011207.

Miller, F. R. (1913) On the reactions of the salivary centers. Q. J. Exp. Physiol. 6: 57-72.

Miyake, Y. (1969) Chorda versus glossopharyngeal nerve in the reflex parotid secretion. J. Physiol. Soc. Jpn. 31: 268-276 (in Japanese).
Norgren, R. (1978) Projections from the nucleus of the solitary tract in the rat. Neuroscience 3: 207-218.

Norgren, R., and C. M. Leonard (1973) Ascending central gustatory pathways. J. Comp. Neurol. 150: 217-238.

Roberts, W. W. (1980) $\left[{ }^{14} \mathrm{C}\right]$ Deoxyglucose mapping of first order projections activated by stimulation of lateral hypothalamic sites eliciting gnawing, eating, and drinking in rats. J. Comp. Neurol. 194: 617-638.

Saper, C. B., A. D. Loewy, L. W. Swanson, and W. M. Cowan (1976) Direct hypothalamo-autonomic connections. Brain Res. 117: 305-312.

Schneyer, L. H., J. A. Young, and C. A. Schneyer (1972) Salivary secretion of electrolytes. Physiol. Rev. 52: 720-777.

Sessle, B. J., and L. F. Greenwood (1976) Inputs to trigeminal brain stem neurons from facial, oral, tooth pulp and pharyngolaryngeal tissues. I. Responses to innocuous and noxious stimuli. Brain Res. 117: 211-226.

Snell, R. S. (1960) The effect of preganglionic parasympathectomy on the structure of the submandibular and major sublingual salivary glands of the rat. Z. Zellforsch. Mikrosk. Anat. 52: 686-696.

Snell, R. S., and J. R. Garrett (1957) The distribution of cholinesterase in the submaxillary and sublingual salivary glands of the rat. J. Histochem. Cytochem. 5: 236-245.

Suzuki, T. (1981) Reflex discharges recorded from rat submandibular ganglion cells in vivo. Bull. Tokyo Dent. Coll. 22: 115-124.

Suzuki, T., and S. Sakada (1972) Synaptic transmission in the submandibular ganglion of the rat. Bull. Tokyo Dent. Coll. 13: $145-164$.

Torvik, A. (1957) Die Lokalisation des "Speichelzentrums" bei der Katze. Z. Mikrosk. Anat. Forsch. 63: 317-326. 